\title{
2D+T Acoustic Boundary Detection in Echocardiography
}

\author{
Miguel Mulet-Parada and J. Alison Noble \\ \{miguel, noble\}@robots.ox.ac.uk
}

Department of Engineering Science. University of Oxford, Oxford OX1 3PJ, UK

\begin{abstract}
In this paper we address the problem of spatio-temporal acoustic boundary detection in echocardiography. We propose a phasebased feature detection method to be used as the front end to higher-level $2 \mathrm{D}+\mathrm{T} / 3 \mathrm{D}+\mathrm{T}$ reconstruction algorithms. We develop a $2 \mathrm{D}+\mathrm{T}$ version of this algorithm and illustrate its performance on some typical echocardiogram sequences. We show how our temporal-based algorithm helps to reduce the number of spurious feature responses due to speckle and provides feature velocity estimates. Further, our approach is intensityamplitude invariant. This makes it particularly attractive for echocardiographic segmentation, where choosing a single global intensity-based edge threshold is problematic.
\end{abstract}

\section{Introduction}

This paper develops a novel technique for finding acoustic boundaries in 2D and $2 \mathrm{D}+\mathrm{T}$ echogram sequences. Most prior work has involved the development of 2D spatial processing methods, based on, for example, template mask feature detection [1]; 'snakes' [2], or integrated backscatter (IBS) boundary detection [3]. However, spatial methods ignore temporal continuity, which can potentially be used to improve the reliability of feature detection. Herlin and Ayache investigated this idea [4]. They proposed tracking cardiac boundaries using a method that employed a spatio-temporal based version of the Deriche edge detector [5] and assumed a Gaussian noise model. However, their approach was not fully developed or validated on clinical data.

Acoustic image feature detection needs to be robust to speckle noise and attenuation imaging artefacts. Speckle noise corrupts the data by introducing sharp changes in the image intensity profile, while attenuation artefacts alter the intensity of equally significant cardiac structures depending on their orientation with respect to the ultrasound beam. This suggests that measures based on phase information rather than intensity derivatives may be more appropriate for acoustic feature detection.

In previous work we have discussed the application of phase-based feature extraction methods to echocardiographic data [7]. We used a local-phase measure to selectively detect step edges associated with cardiac boundaries in 1D echocardiographic profiles. Local phase was computed via the convolution of the image with a quadrature pair of filters [6]. A scalogram was used to show that 
local phase signatures of cardiac boundaries are detectable and well localised at large scales. This is not the case for small scale speckle noise. Further, we showed that step-like cardiac boundaries can be selectively extracted according to the value of their local-phase signature which is different from that of the ridge-like structures associated with speckle. We applied this approach to 1D acoustic boundary detection in a Kalman-filter based tracking algorithm, and showed that this method gives better tracking performance than using simple gradient-based edge detection.

Unfortunately, the technique used in [7] cannot be readily extended to two or more dimensions. Building on the work of Kovesi [8], in the rest of this paper we present a new phase-based measure that shares the good localisation and selectivity properties of local phase but which can be extended to $2 \mathrm{D}$ and $2 \mathrm{D}+\mathrm{T}$. We also show how the $2 \mathrm{D}+\mathrm{T}$ version of this algorithm provides an estimate of feature velocity. We are currently investigating how this idea can provide input to a higher level algorithm.

The outline of the paper is as follows. Section 2 presents the 2D acoustic feature detection algorithm and motivates the need for spatio-temporal analysis. This leads us to propose a $2 \mathrm{D}+\mathrm{T}$ measure in Section 3 and to show how feature velocity can be estimated. Section 4 presents preliminary experimental segmentation results on real $2 \mathrm{D}$ cardiac sequences. We conclude with a summary and a discussion of current and future work in Section 5.

\section{The 2D feature asymmetry algorithm}

Kovesi proposed a 2D multi-scale, intensity-invariant, feature detection measure for finding step features in visual images [8]. In [7] we showed that a simplified version of this algorithm, using a single large scale, gives well localised segmentation results. We call this measure $2 \mathrm{D}$ feature asymmetry, $F A_{2 D}(x, y)$, which we define as,

$$
F A_{2 D}(x, y)=\sum_{m} \frac{\left\lfloor\left[\left|o_{m}(x, y)\right|-\left|e_{m}(x, y)\right|\right]-T_{m}\right\rfloor}{A_{m}(x, y)+\epsilon} .
$$

Here $o_{m}(x, y)\left(e_{m}(x, y)\right)$ is the output of a convolution of an image with an orientable odd (even) symmetric log-Gabor wavelet filter, and the summation is taken over $m$ filter orientations. $A_{m}$ is the local energy amplitude of the $m$ th filter response given by $A_{m}(x, y)=\sqrt{e_{m}(x, y)^{2}+o_{m}(x, y)^{2}}, \downarrow$ denotes zeroing of negative values and $\epsilon$ avoids division by zero. The orientable $2 \mathrm{D}$ filters are defined by "spreading" a log-Gabor function into two dimensions. Namely, an orientable filter tuned to a particular orientation $\phi_{0}$ is constructed in the frequency domain by masking a radial log-Gabor function with an angular Gaussian tuned to $\phi_{0}$,

$$
G\left(\omega_{r}, \phi\right)=\exp -\left(\frac{\left(\log \left(\omega_{r} / \omega_{r_{0}}\right)\right)^{2}}{2\left(\log \left(\kappa / \omega_{0}\right)\right)^{2}}+\frac{\left(\phi-\phi_{0}\right)^{2}}{2 \sigma_{\phi}^{2}}\right) .
$$

Here $\phi_{0}$ is the orientation of the filter and $\sigma_{\phi}$ defines the extent of the spreading function as a scaling $s$ of the separation between filters $\Delta \phi$ i.e. $\sigma_{\phi}=s \times \Delta \phi$. The value $\kappa / \omega$ determines the wavelength of the filters. In our experience we have 
found that $\Delta \phi=30^{\circ}, s=0.6, \kappa / \omega=0.55$ (2 octaves), provides a good compromise between even spectral coverage, orientation resolution and computation time for a six filter bank. The orientation-dependent noise threshold, $T_{m}$, and the zeroing operation make each filter respond to only those features in its orientation range. In Equation 1, amplitude normalisation provides invariance to contrast. Thus, the response of each feature is normalised according to the local energy measure around its neighbourhood. In this way local feature significance prevails over general feature strength.

The result of computing 2D feature asymmetry on a short-axis ultrasound image with no pre-filtering is shown in Figure 2(B). In our images a filter wavelength of 20 pixels has been found to give good feature localisation while avoiding the detection of speckle. The results show good detection of the chamber boundaries. However, the limitation of using a 2D feature asymmetry measure is highlighted if the $2 \mathrm{D}$ (spatially) processed images are displayed as a movie sequence. In this case, a number of small flickering spurious features are observed. In any given frame, there will always be a number of spurious features, due to noise and artefacts, that share similar intensity, shape and scale characteristics as responses from chamber boundaries. However, and unlike cardiac chamber boundaries, most of these features are not persistent from one frame to the next. In fact, most are due to speckle patterns that decorrelate with tissue movement. To remove the flickering noise, we now extend the $2 \mathrm{D}$ analysis into the temporal domain.

\section{The $2 \mathrm{D}+\mathrm{T}$ algorithm and feature velocity estimation}

The extension of the feature asymmetry measure to $2 \mathrm{D}+\mathrm{T}$ involves the design of a set of filters oriented in a number of spatio-temporal directions. This is achieved by re-defining the $2 \mathrm{D}$ filters in $3 \mathrm{D}$ spherical co-ordinates,

$$
G\left(\omega_{r}, \phi, \theta\right)=\exp -\frac{\left(\log \left(\omega_{r} / \omega_{r_{0}}\right)\right)^{2}}{2\left(\log \left(\kappa / \omega_{0}\right)\right)^{2}} \exp -\left(\frac{\left(\phi-\phi_{0}\right)^{2}}{2 \sigma_{\phi}^{2}}+\frac{\left(\theta-\theta_{0}\right)^{2}}{2 \sigma_{\theta}^{2}}\right) .
$$

The velocities to which each filter responds are encoded in terms of $\phi_{0}$ the spatial orientation, and $\theta_{0}$ the temporal orientation. Figure 1 shows a diagrammatic representation of the filter orientations. Twenty-one filters are located on a spatio-temporal hemisphere at the vertices of an 80 faceted tesselation of the unit sphere obtained from the subdivision of a regular icosahedron. This provides an approximately even distribution of filters over the spatio-temporal space. Note that for $\theta=0$ (time $=0$ plane) only orientations in the $\phi=\left[0^{\circ}, 180^{\circ}\right)$ interval are required. The spread parameters are chosen to be the same, making the filters isotropic in the spatial and temporal dimensions $\left(\sigma_{\phi}=\sigma_{\phi}\right)$. The value of the spatio-temporal spread is set to a fraction (0.6) of the average angular spacing between filters to ensure even spectral coverage.

In order to improve the contrast of a segmented image, the maximum filter output over all orientations is taken rather than the sum. The $2 \mathrm{D}+\mathrm{T}$ feature asymmetry measure then becomes, 


$$
\mathrm{FA}_{2 D+T}(x, y)=\max _{v, m} \frac{\left\lfloor\left[\left|o_{m, v}(x, y)\right|-\left|e_{m, v}(x, y)\right|\right]-T_{m, v}\right\rfloor}{A_{m, v}(x, y)+\epsilon}
$$

where the indices $v, m$ respectively indicate the temporal and spatial orientation of each filter.

Note that we do not choose particular velocities but sample over all the spatio-temporal space. The current implementation does not optimise filter design to a velocity range because cardiac motion varies during the cardiac cycle, depends on position on the heart surface, and image velocities ultimately depend on the temporal resolution of the data. In practice, provided that the spatiotemporal filters have sufficient resolution and tile the spatio-temporal volume evenly, any velocity will be detected by at least one filter. The $\theta=90^{\circ}$ filter detects very fast changes that persist over time, i.e. pure temporal steps due to occlusions.

Note further that by recording which of the filters gives the strongest response an estimate of feature velocity is obtained. Given the sparse sampling of the $2 \mathrm{D}+\mathrm{T}$ space the estimate can be improved by interpolating the responses around the maximum to approximate the true feature velocity.

The current implementation performs filter con-

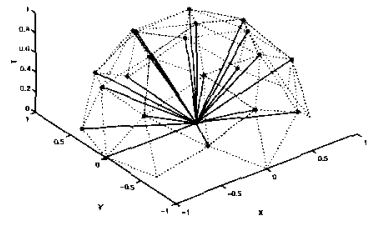

Fig. 1. The 21 $2 D+T$ filter orientations. volutions by multiplication in the frequency domain using Temperton's FFT algorithm [9]. The filters are built in the frequency domain using Equation 3. The separability of the 3D FFT operation allows us to resample the filter's spectrum according to the relative data lengths of each axis in space-time. We treat the $2 \mathrm{D}+\mathrm{T}$ volume to be isotropically sampled. In the results presented in Section 4 the data dimensions are $256 \times 256$ pixels in space by 32 frames in time.

\section{Results}

In this section we present the results of applying our $2 \mathrm{D}+\mathrm{T}$ acoustic boundary detection algorithm to a number of echogram sequences. We illustrate some of the distinguishing features of this type of approach to acoustic feature detection and highlight some potential areas of application of the technique. We encourage the reader to visit http://www.robots.ox.ac.uk/ miguel/MICCAI98.html where the movies referred to in this section and further examples can be seen.

4.1 Comparing 2D and 2D+T filters: The purpose of the first experiment is to highlight some of the differences between $2 \mathrm{D}$ and $2 \mathrm{D}+\mathrm{T}$ boundary detection. A 2D+T filter bank, consisting of 21 filters, was designed as described in Section 3. The wavelength of the Log-Gabor component was 20 pixels for all the filters, their bandwidth was 2 octaves, $\kappa / \omega_{0}=0.55$, and $s=0.6$.

Three consecutive frames $(11,12$ and 13 in the movie sequences) of the $2 \mathrm{D}+\mathrm{T}$ segmentation are shown in Figure 2(C). For comparison we have included the original data and the results of a $2 \mathrm{D}$ segmentation using the same scale, Figures $2(\mathrm{~A})$ and $2(\mathrm{~B})$ respectively. 
Note the absence of small scale flicker features in the $2 \mathrm{D}+\mathrm{T}$ measure when compared with the $2 \mathrm{D}$ result. The significant features in the $2 \mathrm{D}+\mathrm{T}$ results can be easily traced across the three frames and they all correspond to relevant cardiac structure. In the 2D segmentation this is not the case. Many inconsistent bloblike features are detected as well as a number of short-lived small-scale line features corresponding to speckle. Furthermore, in the $2 \mathrm{D}+\mathrm{T}$ result the left wall of the ventricle is visible as a faint arc. This structure is not detected by $2 \mathrm{D}$ segmentation. The results illustrate how the $2 \mathrm{D}+\mathrm{T}$ segmentation weighs the importance of spatial features according to their temporal significance.

With larger filter scales, smoother contours are obtained and less noise is detected, but this is at the price of less accurate localisation and detail. Obviously, increasing the scale of the 2D filters does not remove flicker noise, (see the web page for an illustration of this). In Figure 3(A) we show 2D and 2D+T results on a very cluttered long axis image. This is the other standard view used to quantify left ventricle dynamics by cardiologists. The results show very clearly the removal of the flickering speckle features and the enhancement given to the main cardiac structures by $2 \mathrm{D}+\mathrm{T}$ filtering.

A limitation of our current $2 \mathrm{D}+\mathrm{T}$ algorithm is that it cannot detect fast cardiac motions that have similar temporal characteristics to speckle; for example the mitral valve leaflets. At typical diagnostic frame rates $(\sim 50 \mathrm{~Hz})$ the $2 \mathrm{D}$ segmentation of the mitral valve appears superior to the $2 \mathrm{D}+\mathrm{T}$ results because, during opening, the leaflets cannot be tracked consistently across time frames. This is illustrated in Figure 3(B). The whole sequences can be seen in the web page. Note that this is not a problem of the algorithm itself but of the limited sampling rate of the data. This can be overcome by using faster frame rates (as available on new commercial machines) or interpolating the sequence prior to detection.

4.2 Gradient vs. phase-based filtering: The full spatio-temporal character of our method and its invariance to contrast, are illustrated in this section by comparison with a gradient-based spatio-temporal algorithm developed by Herlin and Ayache [4].

Herlin and Ayache used a modification of Deriche's edge detector in 3D based on smooth derivatives [5]. They estimated the two spatial components of the gradient vector $N_{x}$ and $N_{y}$ from the convolution of the $2 \mathrm{D}+\mathrm{T}$ intensity function $I(x, y, t)$ with two spatial derivative kernels $\left(D_{x}, D_{y}\right)$ and a temporal smoothing filter $\left(L_{t}\right)$ so that $N_{x}=\left(D_{x}, L_{y}, L_{t}\right) \otimes I(x, y, t)$, and, $N_{y}=\left(L_{x}, D_{y}, L_{t}\right) \otimes$ $I(x, y, t)$. Edges were obtained from the local maxima of the norm of the gradient given by, $N(x, y, t)=\sqrt{N_{x}^{2}+N_{y}^{2}}$.

The result of applying the Deriche method to our short axis sequence is shown in Figure 3(C). The same parameters as in [4] were used. The Deriche method is contrast dependent and features are detected based on the size of intensity derivatives rather than on their spatio-temporal significance and shape. The result is a cluttered edge strength image where relevant and irrelevant features are detected with similar strength. Further, the Deriche method is not truly spatio-temporal. The derivation is only performed in the spatial domain and the 
temporal filtering only contributes by smoothing the image over time, not by detecting temporal features.

4.3 Velocity measurements: Finally, we present results of feature velocity estimation. Velocity is estimated from the inclination of the filter exhibiting the strongest response. As the cardiac muscle gains speed during systole, wall features in the spatio-temporal domain are detected by faster moving filters (larger $\theta_{0}$ ). At end-systole the muscle velocity decreases to zero before diastolic relaxation and the features are detected by static filters in the spatial plane $\left(\theta_{0}=0\right)$. Colour coded response maps for a series of views can be seen at our web page. Figure 3(D) shows two frames during systole and diastole. Arrows have been marked on the detected features after non-maximal suppression of the feature asymmetry image. In this case the orientation and magnitude of these arrows corresponds to the velocity vector estimated from the interpolated filter responses around the maximum. We are currently investigating how these measurements can provide input to an active-contour based tracker. Current research is also comparing this approach to velocity estimation using Doppler Tissue Imaging [11].

\section{Discussion}

We have presented a new approach to spatio-temporal acoustic boundary detection based on phase-based methods. The main contributions of this work are (1) to propose a new phase-based scheme for detecting acoustic boundaries in echocardiographic image sequences and (2) to develop a $2 \mathrm{D}+\mathrm{T}$ version of this algorithm that is resistant to temporally inconsistent speckle and that can provide a measure of feature velocity. A key advantage of this approach is that it is intensity-amplitude invariant. This makes it especially attractive for echocardiographic $3 \mathrm{D}+\mathrm{T}$ reconstruction where choosing a single global intensity-based edge threshold is problematic due to position dependent attenuation.

We are currently using our phase based segmentation and velocity estimation as the front end to a higher level interpretation system based on a snake framework similar to our work in [10,7], and also plan to extend our ideas to volumetric spatio-temporal data. We also plan to perform a more detailed study of filter design and filter response interpolation in order to improve the accuracy of feature velocity estimation. Quantitative assessment and evaluation against related measurement protocols, particularly Doppler Tissue Imaging will also be performed. Finally, an interesting variant of this work might be to investigate the use of a symmetry measure for speckle tracking.

\section{References}

1. D.C. Wilson, E.A. Geiser, and J-H. Li. Feature extraction in two-dimensional short-axis echocardiographic images. J. of Math. Imag. and Vis., 3:285-298, 1993.

2. V. Chalana, et al. A multiple active contour model for cardiac boundary detection on echocardiographic sequences. IEEE Trans. Med. Imag., 15(3):290-298, 1996.

3. A. Lange, et al. The variation in integrated backscatter in human hearts in differing ultrasonic transthoracic views. JASE, pages 830-838, Nov-Dec 1995. 
4. I. Herlin and Ayache.N. Feature extraction and analysis methods for sequences of ultrasound images. In ECCV92, pages 43-57, 1992.

5. R. Deriche. Fast algorithms for low-level vision. PAMI-12(1):78-87, January 1990.

6. S. Venkatesh and R.A. Owens. An energy feature detection scheme. In ICIP98, pages 553-557, Singapore, 1989.

7. G. Jacob, A. Noble, M. Mulet-Parada, and A. Blake. Evaluating a robust contour tracker on echocardiographic sequences. MedIA Journal, to appear 1998.

8. P. Kovesi. Invariant measures of image features from phase information. PhD thesis, University of Western Australia., 1996.

9. C. Temperton. A generalised prime factor FFT algorithm for any $n=2^{p} \times 3^{q} \times 5^{r}$. SIAM J. Sci. Stat. Comp., May 1992.

10. G. Jacob, A. Noble, and A. Blake. Robust contour tracking in echocardiographic sequences. In ICCV98, Bombay, India, pages 408-413, January 1998.

11. M.A. Garcia Fernandez et al.. Doppler Tissue Imaging Echocardiography. McGraw-Hill, Madrid 1998.

A.
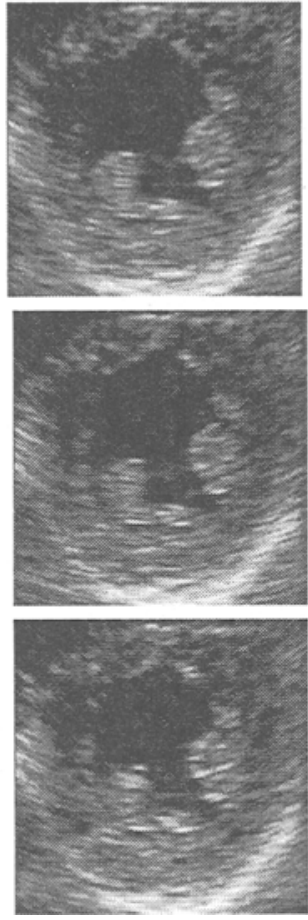
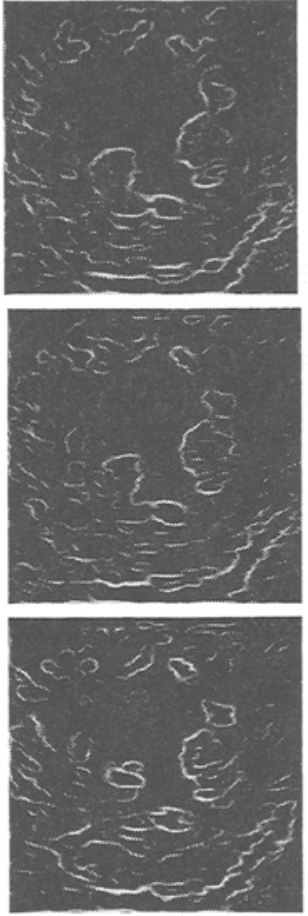
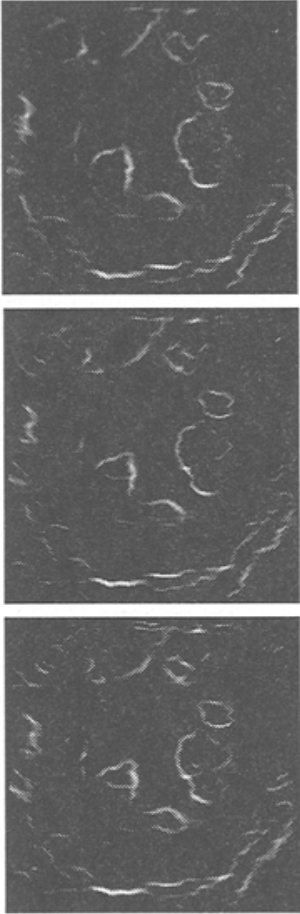

Fig. 2. Comparison of $(A)$ input frames, (B) $2 D$ feature asymmetry results, (C) $2 D+T$ feature asymmetry results. 
A.

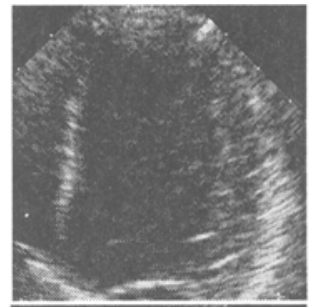

B. 1

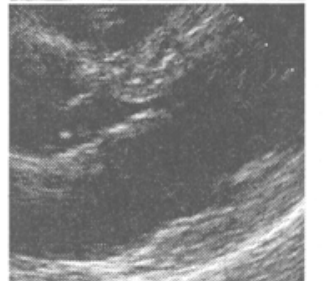

1

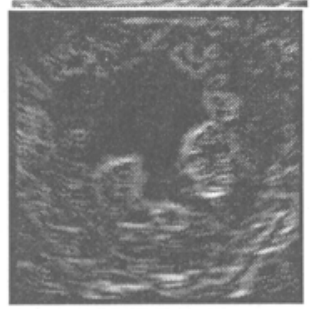

B. 2
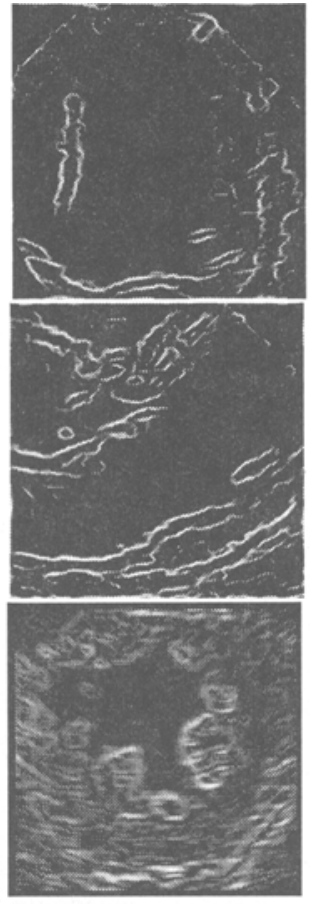

B. 3
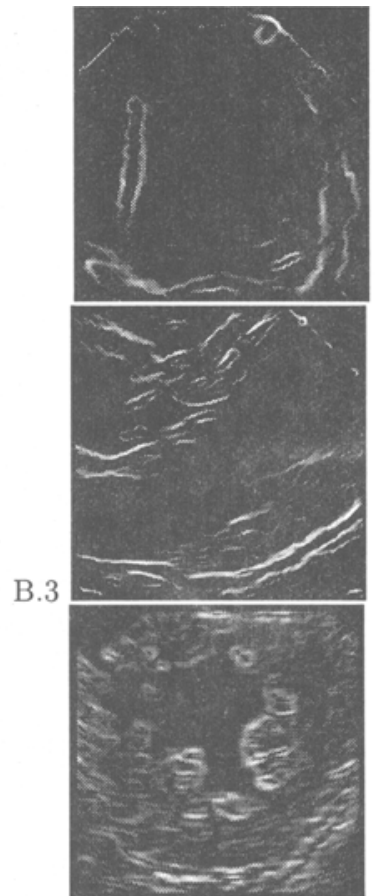

C

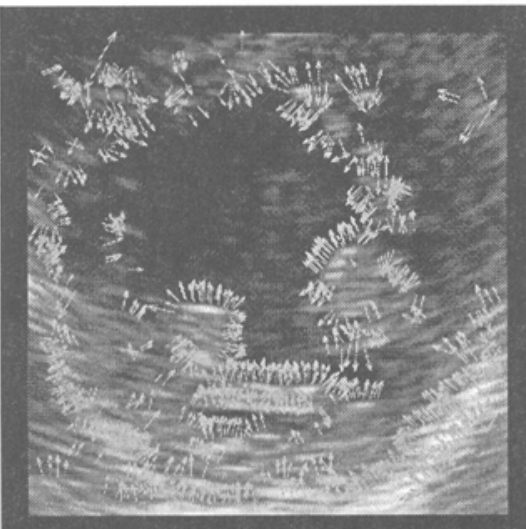

D.1

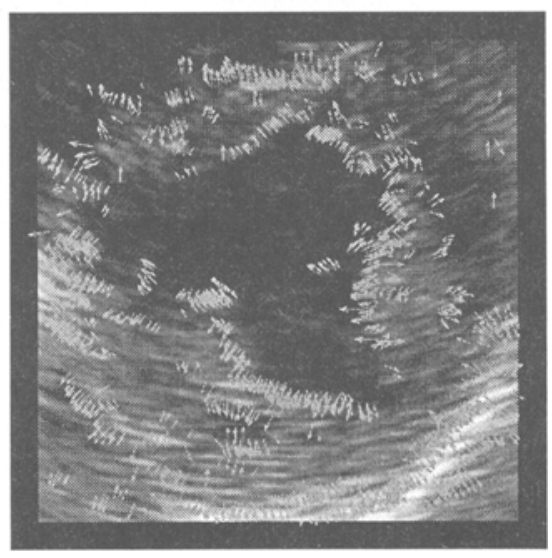

Fig. 3. (A) Long axis frame, 2D and 2D+T feature asymmetry results. (B) Valve Results. (1) View of the Mitral and Aortic Valves at opening. (2) $2 D$ and (3) $2 D+T$ feature asymmetry results (C) Results of running Herlin and Ayache's method on our data. (D) Interpolated fitter response velocity estimates, (1) systole: arrows point inwards, (2) diastole: arrows point outwards. 